\title{
COMPUTATION OF LYAPUNOV EXPONENT FOR Characterizing THE DYNAMics OF EARTHQUAKE
}

\author{
Folasade. L. Aderemi and Olatunde. I. Popoola \\ ${ }^{1}$ Department of Physics, University of Ibadan, Ibadan, Nigeria.
}

\begin{abstract}
Earthquakes forecasting and prediction is a global challenge, several precursors and methods of earthquake prediction have been proposed, but lack consistency and are not reliable for prediction hence occurrence of earthquakes is sometimes assumed to be random. This study was designed to investigate and characterise the occurrence of earthquakes using chaos theory. The Lyapunov Exponent and its spectrum were obtained from earthquake data using modified two dimensional system method from Sprott's procedures. The results show that the values of the Lyapunov exponent were positive but the magnitude varies for all regions considered and the Lyapunov exponent spectrum exhibit an asymptotic behaviour in all the regions.This study showed that although seismicity exhibit apparent randomness but earthquake occurrence is not stochastic but a non-linear deterministic dynamical process.
\end{abstract}

\section{KEYWORDS}

Earthquake prediction, non-linear dynamic, chaos theory, computation,Lyapunov exponent

\section{INTRODUCTION}

Earthquake can be defined as a sudden release of accumulated strain energy in a confined region of the Earth interior or along a fault or fracture in the earth's crust, The strain is the result of stress which occurs along plate boundaries due to the relative motion of the lithospheric plates.Earthquake prediction is still a global challenge because, the hypocenters of earthquakes are inaccessible and the state of the lithosphere at seismogenic depth can not be observed directly [1]. The earth crust where most earthquakes occur is highly heterogeneous in distribution of strength and stored elastic strain energy. No satisfactory theory of earthquake source process exists at present [2]. Application of dynamics theory is limited because response of rocks to stress is highly complex and non linear. The interaction of faults is highly complex and non linear. The relationship is complex and may differ in seismogenic zones [3]. Laboratory and experimental models are conducted on a limited scale and do not replicate the complex and heterogeneous conditions of the problem or phenomenon in situ. Determining the history of earthquakes is difficult in some areas because the historic record of such area is short compared with the average time between major earthquakes [4]. Because little is known about the physics of faulting many attempts to predict earthquakes have searched for precursors [5].Several precursors have been proposed such as: Foreshocks,Seismic cycle, Seismic Doughnut, Seismic quiescence [5]. Radon concentration and temperature decrease in groundwater; Groundwater rise in well [6,7]. However

DOI : 10.14810/ijrap.2016.5402 
these precursors do not reproduce themselves [8-10]. Lack of consistency in seismic precursors and inability to predict earthquake has made many seismologists to conclude that earthquake occurrence is stochastic and its prediction is a Gambler's fallacy[11] Occurrence of earthquakes is assumed to be random when these methods produce unreliable pattern[12-15]. The fact that these patterns considered to be random could be chaotic (predictable but difficult) has not been investigated. For earthquake to be predicted or predictable there is a need to develop a physics based theory of the seismogenesis, nucleation and precursory process of the earthquake. The dynamic nature of earthquake must be clearly understood in a scientific manner andmethod, not just by speculation hence there is the need to know and not assumed whether the occurrence of earthquake is periodic, chaotic or random in space and time.This study was designed to investigate and characterized the dynamics of earthquakes' occurrence using chaos theory.

\section{BACKGROUND}

A stochastic or random process is a probabilistic process as opposed to a deterministic process because there is some indeterminacy such that even if the initial condition or starting point is known, there are several many directions in which the process may evolve. A similar initial condition may give entirely different result or output.In science it is a non-linear dynamical system that lies between regular deterministic system and stochastic system. A chaotic system is deterministic but difficult to predict because it is sensitive to initial condition (the butterfly effect).

\subsection{ChaOTIC System}

Chaos is a type of motion that lies between regular deterministic trajectory that are predictable and the unpredictable stochastic behaviour that is characterized by complete randomness, Nonlinear dynamical systems that appear to have random, unpredictable behaviour, in other words the chaotic system follows deterministic rule but its evolution appear random[16]. Chaotic system is a dynamic system that is deterministic, non-linear, sensitive to initial condition hence difficult to predict or a stochastic behaviour occurring in a deterministic system. Chaos is the study of deterministic systems that are so sensitive to measurement that their output appears random [16, 17].

\subsection{LYAPUNOV EXPONENT}

The Lyapunov exponent is a quantitative measurement of the rate of exponential divergent or convergent of a dynamic system and it is a basic indicator of deterministic chaos [18].

The sign of the Lyapunov exponents provide a qualitative picture of a system's dynamics. Its sign indicates whether the system is chaotic or not. The magnitude of the Lyapunov exponent reflect the time scale in which the system dynamic become unpredictable while its sign indicates sensitivity to initial condition[19,20].The Lyapunov exponents quantify the exponential divergence of initially close state-space trajectories and estimate the amount of chaos in a system[21]. The Lyapunov exponent is positive when neighbouring trajectories diverge from each other at large n, which corresponds to chaos. However, if the trajectories converge to a fixed point or limit cycle, they will get closer together, which corresponds to negative Lyapunov exponents. Hence we can determine whether or not the system is chaotic by the sign of the Lyapunov exponent. It is a way of distinguishing between a stochastic process and a deterministic system[22].Obtaining the Lyapunov exponents from a system with known 
differential equations is no real problem but difficult or nearly impossible for experimental data without known differential equations[20].Experimental data typically consist of measurements of a single observable. There is a need to employ reconstruction and locating the nearest neighbour on the trajectory. The nearest neighbour is at a minimal point or distance from the reference point.

\section{MAterial ANd Method}

\subsection{DATA ACQUiSition}

The data used in this study were obtained from Earthquake catalog of Advanced National Seismic System (ANSS), Northern California Earthquake Data Centre, and USA, Period of catalogue 1899-2009. The catalog contain the origin time, date, epicentre, depth in kilometre, latitude , longitude, magnitude and magnitude type

\subsection{METHODS}

Locations and magnitudes of large earthquakes globally were identified from 1899 to 2009. Large earthquake in the circum-pacific zone were mapped and divided into three regions: Region 1 is within Latitude $48^{\circ}$ to $60^{\circ}$ and Longitude $-179^{\circ}$ to $-160^{\circ}$, Region 2 is within Latitude $32^{\circ}$ to $44^{\circ}$ and Longitude $134^{\circ}$ to $148^{\circ}$ while Region 3 is within Latitude $-42^{\circ}$ to $-29^{\circ}$ and Longitude $-80^{\circ}$ to $-66^{\circ}$.The Lyapunov exponent and spectrum were computedbased on the following procedure. If two orbits are separated by a small distance $d_{o}$ at time $t=0$, then at a later time $t$, their separation is given by

$d(t)=d_{0} e^{\lambda t}(1)$

If the system evolves through an iteration process then

$d_{n}=d_{0} e^{\lambda n}(2)$

Where $\mathrm{n}$ is the number of iteration hence $\lambda$ is dimensionless.

$$
\begin{aligned}
& \lambda_{n}=\frac{1}{n} \sum_{j=1}^{n} \ln \left|\frac{d_{j}}{d_{o}}\right|, n \neq 0(3) \\
& d_{j}=\sqrt{\left(\left(x_{a}-x_{b}\right)^{2}+\left(y_{a}-y_{b}\right)^{2}\right)} \\
& d_{0}=\sqrt{\left(\left(x_{a}-x_{b o}\right)^{2}+\left(y_{a}-y_{b o}\right)^{2}\right.}
\end{aligned}
$$

For a two dimensional system with variables $\mathrm{x}$ and $\mathrm{y}$, the separation is defined by equation 4 where $a$ and $b$ denote the two orbits [23].If $a$ and $b$ denote two orbits, $d_{j}$ is defined as the separation between the $\mathrm{j}^{\text {th }}$ pair of nearest neighbours while $\mathrm{d}_{\mathrm{o}}$ is the separation between the first pair of nearest neighbours, this is also known as the initial separation. In this study, since 
earthquake is a single observable, $\mathrm{x}$ is defined as the latitude while $\mathrm{y}$ is defined as the longitude of epicentre of the earthquake. $x_{a}$ and $y_{a}$ are defined as the latitude and longitude of a referenced large earthquake respectively while $\mathrm{x}_{\mathrm{b}}$ and $\mathrm{y}_{\mathrm{b}}$ are the latitude and longitude of the subsequent earthquakes respectively. The initial pair of nearest neighbours are the referenced large earthquake and the first subsequent earthquake (in time and space) within the region hence $d_{o}$ is defined as the initial separation between the first subsequent earthquake and the reference large earthquake while $d_{j}$ is the separation between the $j^{\text {th }}$ subsequent earthquake and the reference large earthquake, this is necessary because earthquake is a single observable

\section{RESULTS AND DiSCUSSION}

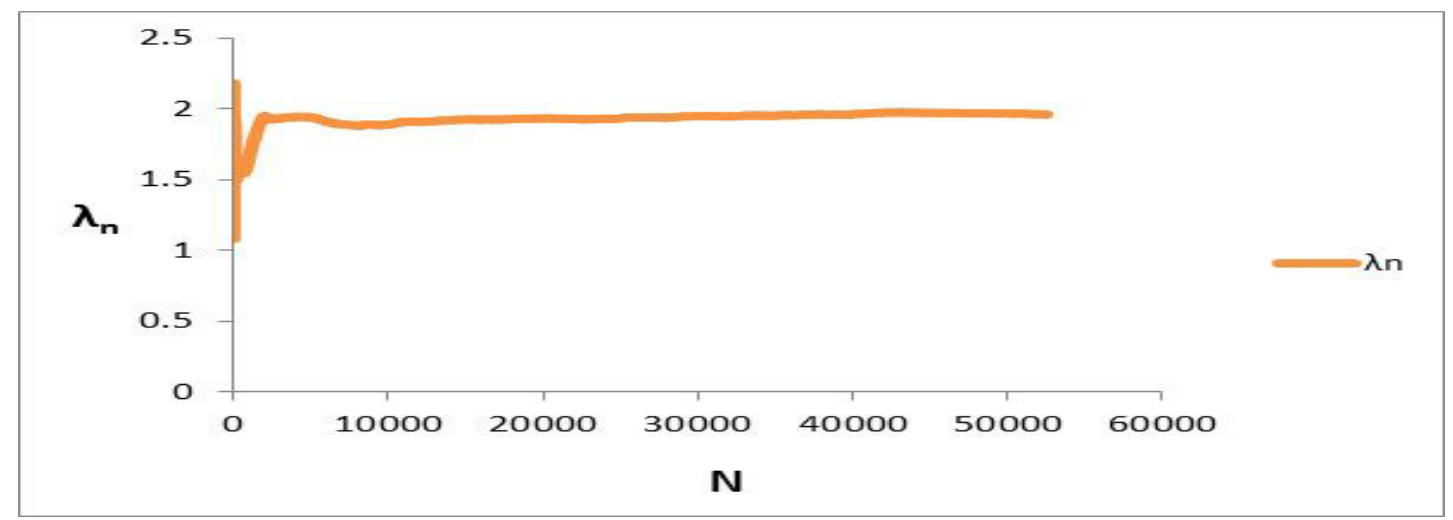

Figure 1; Lyapunov exponent spectrum for Region 1

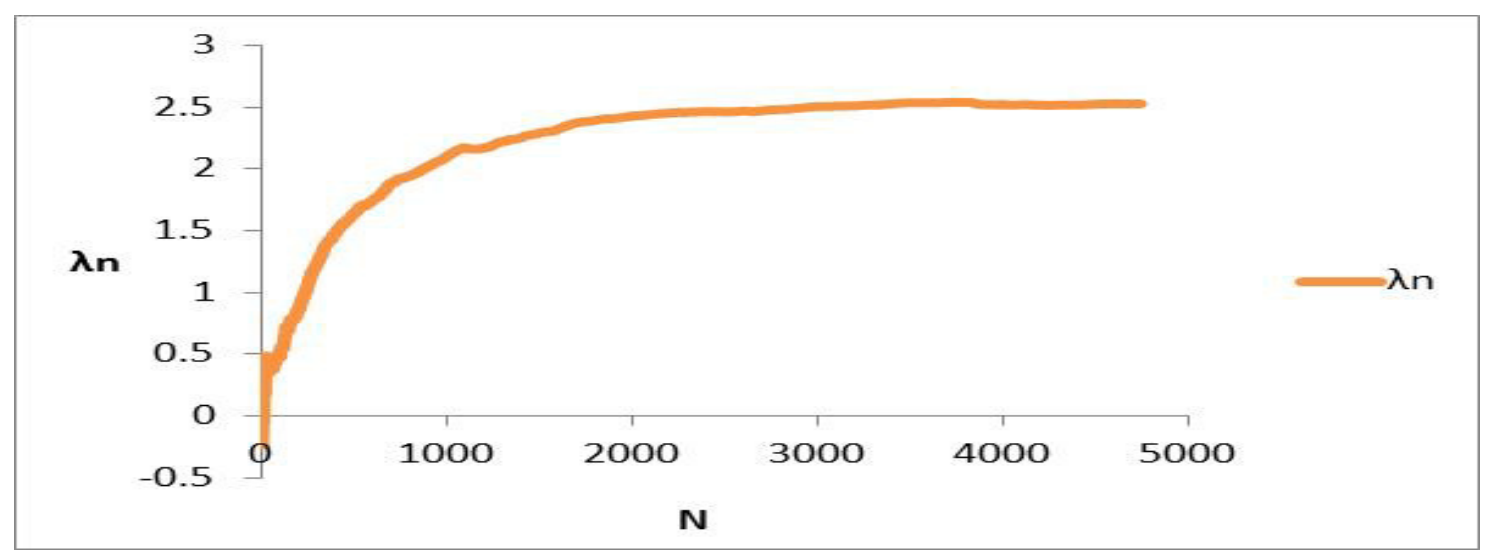

Figure 2. Lyapunov exponent spectrum for Region 2 
International Journal of Recent advances in Physics (IJRAP) Vol.5, No.3/4, November 2016

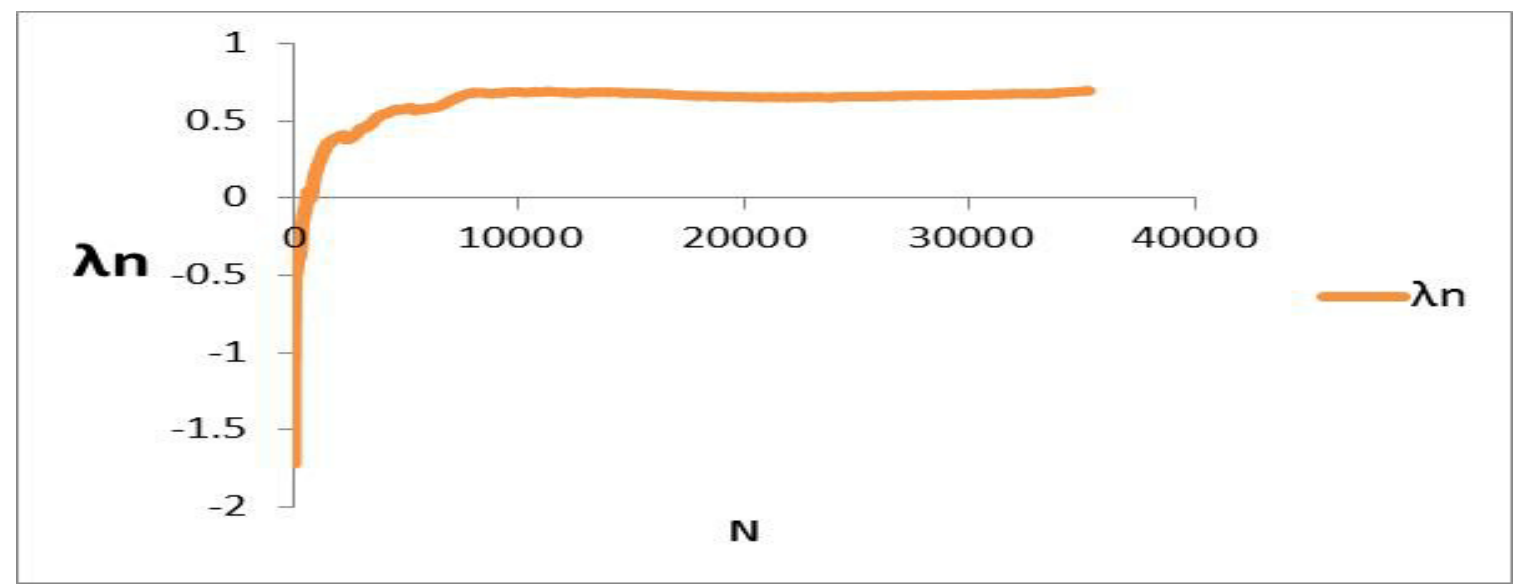

Figure 3. Lyapunov exponent spectrum for Region 3

Table 1: the Lyapunov exponents for the three regions

\begin{tabular}{|cc|}
\hline Region & LEX \\
\hline 1 & 1.963 \\
2 & 2.688 \\
3 & 0.688 \\
& \\
\hline
\end{tabular}

The Lyapunov exponent is positive when neighbouring trajectories diverge from each other at large $\mathrm{n}$, which corresponds to chaos. However, if the trajectories converge to a fixed point or limit cycle, they will get closer together, which corresponds to negative Lyapunov exponents. Hence we can determine whether or not the system is chaotic by the sign of the Lyapunov exponent. It is a way of distinguishing between a stochastic process and a deterministic system [22].The Lyapunov exponents quantify the exponential divergence of initially close state-space trajectories and estimate the amount of chaos in a system [21]. It is a basic indicator of deterministic chaos [18].Figure 1 showed the spectrum of the Lyapunov exponent for region 1, Observation showed that the Lyapunov exponent is very sensitive to discontinuity in the catalogue and exhibits an asymptotic behaviour before the occurrence of a large earthquake. Lyapunov exponent spectrum for region 2and region 3 are shown in Figures 2 and 3 respectively, the Lyapunov exponent spectrum exhibit an asymptotic behaviour in all the regions. Table 1 showed the result of the 
Lyapunov exponent computed for the all the regions. The value of the Lyapunov exponent is positive for each of the region but its magnitude varies from one region to another. The lowest value of 0.688 was obtained in Region 3 while the highest value of 2.688 was obtained in Region 2.

\section{CONCLUSION}

The Lyapunov exponent using two-dimensional system method was positive for all the regions, indicating the chaotic nature of the earthquakes occurrence. This was highest for Region 2 (2.688) and lowest for Region 3 (0.688). The Lyapunov exponent spectrum behaved asymptotically prior to the occurrence of the large earthquake, these can serve as a precursor for earthquake forecasting in hazard management.

\section{ACKNOWLEDGEMENTS}

Advanced National Seismic System (ANSS), Northern California Earthquake Data Centre, USA, for earthquake catalogue.

\section{REFERENCES}

[1] Kossobokov, V. (2009) Practise of predicting large earthquake on global and regional scales. Advance School of Non -Linear Dynamics and Earthquake prediction. Trieste, Italy.

[2] Geller, R. J. (1997) Earthquake prediction: A critical review. Geophysical Journal International. 131, 425-450, doi:10.1111/j.1365-246X.1997.tb06588.x.

[3] Biagi, P. F. (1999) Nature Debate, Nature@ Macmillan Publishers Ltd 1999 Registered No. 785998 England.

[4] Michael, A. 1999a. How well can we predict earthquakes? United States Geological Survey, Menlo Park, California, USA. Nature Debate, Nature@ Macmillan Publishers Ltd 1999 Registered No. 785998 England.

[5] Stein, S. and Wysession, M. (2003) An Introduction to Seismology, Earthquakes and Earth Structure. Blackwell Publishing, Ltd.

[6] IASPEI, (1994) International Association of Seismology and Physics of the Earth's Interior, Preliminary list of Significant Precursors.

[7] Roeloffs, E. and Langbein, J. (1994)The earthquake prediction experiment at Parkfield, California, Reviews of Geophysics. 32, 315-335.

[8] Roeloffs, E. and Quilty, E. (1997) Water level and strain changes preceding and following the August 4, 1985 Kettleman-Hills California earthquake. Pure and Applied Geophysics. 149, 21-60.

[9] Scholz, C.H. (1990)The Mechanics of Earthquakes and Faulting.Cambridge University Press.

[10] Wyss, M. (1991) Second round of evaluation of proposed earthquake precursors, Pure and Applied Geophysics 149, 3-16

[11] Campbell, W.H. 1998. A misuse of public funds: UN support for geomagnetic forecasting of earthquakes and meteorological disasters.Eos Trans. Am. Geophys. Union 79, 463-465.

[12] Geller, R. J. Jackson, D. D. Kagan, Y. Y. and Mulargia, F. (1997) Earthquakes cannot be predicted. Science. 275, 1616-1617.

[13] Geller R.J. (1999) Earthquake Prediction: What should we be debating? Nature Debate, Nature@ Macmillan Publishers Ltd 1999 Registered No. 785998 England.

[14] Michael, A. 1999b. Realistic predictions: are they worthwhile? United States Geological Survey, Menlo Park, California, USA Nature Debate, Nature@ Macmillan Publishers Ltd 1999 Registered No. 785998 England. 
International Journal of Recent advances in Physics (IJRAP) Vol.5, No.3/4, November 2016

[15] Worth, R. 2005. The San Francisco Earthquake. Facts On File Incorporated (Environmental Disasters) New York, ISBN 0-8160-5756-7, pg 70-72.

[16] Goldstein, H. Poole, C. and Safko, J. (20020Classical Mechanics. Pearson Education, Inc., publishing as Addison Wesley. ISBN; 0321-188977

[17] Turcotte, D.L. 1991. Fractals and Chaos in Geology and Geophysics. Cambridge University Press.

[18] Sano, M. and Sawada, Y. 1985. Measurement of the Lyapunov spectrum from a chaotic time series. Physics Review Letter. 55 1082-5.

[19] Shaw, R. 1984. The Dripping Faucet as a Model Chaotic System. Santa Cruz, CA: Aerial..

[20 ] Wolf, A. Swift, J. B. Swinney, H.L. and Vastano, J.A. 1985. Determining Lyapunov exponents from a time series, Physica D 16, 285.

[21 ] Rosenstein, M. T. Collins, J. and. De Luca, C. J. 1993. A practical method for calculating largest Lyapunov exponents from small data sets, Physica D 65 (1993) 117-134 North-Holland SDI: 01672789(92)00033-6

[22] Lacasa, L. and Toral, R. 2010. Description of stochastic and chaotic series using visibility graphs, Physical Review, DOI: 10.1103/PhysRevE.82.036120.

[23] Sprott, J.C. 2003. Chaos and time-series analysis. Oxford University Press. ISBN 0-19-850839-5. 